\title{
Illegal Network Identification Optimization Based on Convolutional Neural Network
}

\author{
Weibin Chen ${ }^{\text {a) }}$, Jinhua Yang b) \\ International School, Beijing University of Posts and Telecommunications, Beijing 100876, China. \\ a) chenweibin@bupt.edu.cn \\ b) yangjinhua123@bupt.edu.cn
}

\begin{abstract}
Convolutional neural network (CNN) was a widely used algorithm for image classification in the field of computer vision. At present, in terms of identification of illegal web pages, the main application methods rely on manpower too much, which is a costly and time-consuming method. This paper will apply the CNN algorithm to the identification of illegal networks, build a CNN algorithm framework on the server side of browsers and web pages. It can also use CNN's outstanding performance in image classification to classify images of illegal websites and conducts realtime data on illegal websites. This paper will introduce the CNN algorithm characteristics from the algorithm structure and function.
\end{abstract}

Key words: Illegal Website, Convolutional Neural Network, Image Classification, Algorithm Structure, Application Analysis.

\section{INTRODUCTION}

The identification of these illegal websites is of great significance. However, the number of illegal networks is numerous and continues to increase. Screening these websites by human is very inefficient and often missed. And the recognition technology in the past is not precise enough to be effectively screened. So how to improve the efficiency and accuracy of illegal network identification is critical. At present, the content of illegal websites is mostly picture, which improves the accuracy of image recognition and thus provides a key breakthrough for the identification of illegal websites [1]. With the development of artificial intelligence, it has become possible to identify illegal networks by training machines. Through deep learning, the machine can efficiently identify illegal websites by capturing picture features after the training of a large number of illegal pictures [2].

This paper proposes to use convolutional neural network to establish a screening mechanism on the server side. Using convolutional neural network can identify a large number of illegal websites and update the database in real time. This will save a lot of time and human resources.

From the structural point of view, this paper first introduces the traditional method of identifying illegal networks and analyzes the advantages and disadvantages of these methods. After introducing the convolutional neural network technology, the core functions of the technology are introduced, and the application of the technology to the identification of illegal networks is discussed, and the feasibility, advantages and disadvantages are obtained.

\section{LITERATURE REVIEW}

In the past, the identification of illegal networks was mainly divided into two types. One was manual auditing, and the other was through software. This section will give examples to introduce these two methods. 


\section{Manual Censor}

Manual censor need to employ large number of personnel to specialize in the identification of illegal websites, which has very strict requirements to practitioners. The practitioners need to have extremely high psychological quality and physical strength to identify a large number of illegal information.

\section{Software Monitor}

Software monitor is using software to monitor the content browsed by the browser on the user's browser side. For instance, "Green Dam" is filtering software of the Chinese government's engineering. It mainly uses the technology of image recognition to recognize illegal websites. Its image recognition technology based partly on Open's Hear classifier for face recognition [3]. The working principle is to obtain the image data, normalize the size of the image, separate the skin color and the non-skin color area, then extract the skin color area, and use the proportion of the human face to determine whether it is an erotic image.

\section{APPLICATION OF CONVOLUTIONAL NEURON NETWORK}

In this section, this paper will introduce the history of CNN development and its architecture.

\section{The Development of CNN}

The convolutional neural network originated in 1962.Hubel and Wiesel proposed the concept of the receptive field by studying the visual cortex cells of the cat. The receptive field is a sub-area of the sensitive visual input space of cells of the visual cortex, which make up the entire visual area. Throughout the visual area, cells are divided into two types, simple cells and complex cells [4].

Then 1984 Fukushima put forward the concept of neurocognitive machine based on the receptive field, which is also the predecessor of the convolutional neural network [5]. The so-called visual recognition machine models the visual system, to divide the visual reception into multiple sub-modes, and processes the received image in each progressive feature plane.

Based on neurocognitive machines, CNN was proposed by Yann LeCun in New York University in 1998 [6]. This also marks that the convolutional neural network formally became an important component of current computer vision.

\section{Architecture of CNN}

$\mathrm{CNN}$ is a multi-layer neural network used to process two-dimensional data, such as a black box. The parameters of the black box are set in advance, to initialize the weight of the network with the minimum random number [6]. After that, a large number of images are used as input to the network to train the network. The forward matrix is used to transfer the input through a step-by-step transformation to the output layer, and the backward propagation algorithm compares the output of the network with the corresponding ideal output to adjust the weight matrix. Repeat this two-step training to reach the optimization of network, obtain the mapping relationship between input and output, and then obtain the characteristics of a type of image (convolution kernel).

The internal structure of the black box is similar to the biological neural network. The structure of CNN is divided into input layer, convolution layer, pooling layer, full connection layer and output layer. Neuron nodes between layers are locally connected by using the correlation of local spaces between layers. Here takes the typical LeNet-5 network as an example. Its structure is shown in figure 1 . 


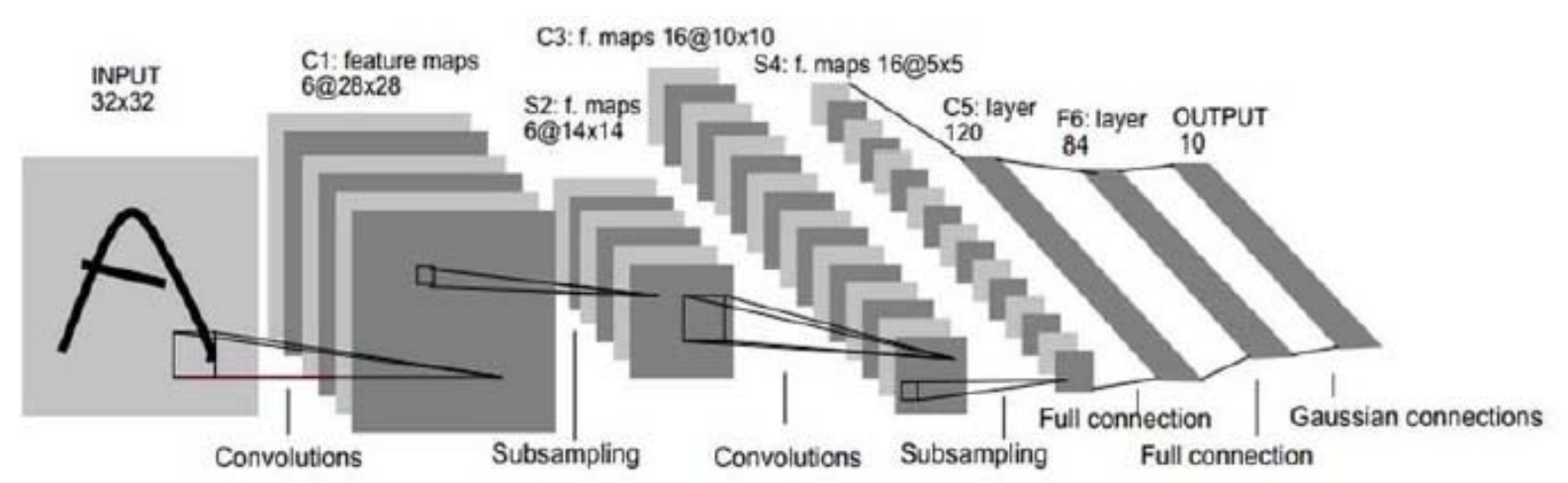

FIGURE 1. The architecture of LeNet-5 network [6].

\section{Convolutional Layer}

The convolutional layer is the main part of the convolutional neural network and is also called the feature extraction layer. At this level, the input pixels are convoluted. The convolution operation can enhance the features of the original signal and reduce interference. In general, multi-core convolutions are used in different convolutional layers to learn different features and extract image features.

Each layer consists of multiple maps. Each map consists of multiple neural units. The neurons in the same map use the same convolution kernel. The input of each neuron in the convolutional layer is connected to the adjacent upper neuron node in the previous layer. By locally perceiving the image, it reduces the number of required weights. In the convolution process, each neuron uses the same convolution kernel to convolute the entire receptive field and extract a feature.

\section{Pooling Layer}

In the pooling layer, the principle of the image local correlation is mainly used to sub-sample the image, which can reduce the amount of data processing while retaining key information. Sample the input feature map, reduce the dimensions of the feature map and extract the main features. In addition, it provides translational rotation invariance. Even if the image is translated or rotated by several elements the result will be same, thereby it improves the accuracy. The common pooling method has max pooling. Its basic principle is to extract the maximum eigenvalues of the relevant pixels in a receptive field, and to sliding sample the pixels of the entire window according to a certain stride. As shown in figure 2[7].

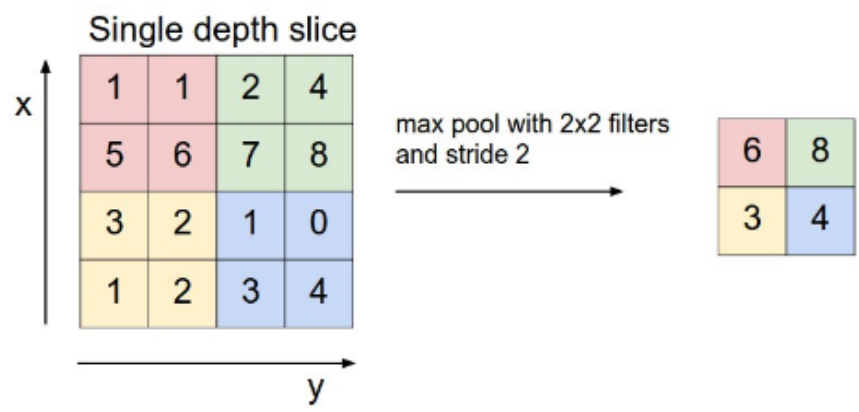

FIGURE 2. The process of Max-pooling [7].

\section{Fully Connected Layer}

The fully connected layer is generally at the end of the convolutional neural network. The fully connected layer connects each node with all nodes on the previous layer. Connect all the features and send the output values to the classifier. The fully connected layer implements the final process of classification in the entire neural network. For 
its connected classifier, there are usually two kinds of classifier, Support Vector Machine or SoftMax. Both of these classifiers are linear classifiers that can linearly classify the selected feature vectors from previous levels. However, it will cause the full connection to contain many complex parameter weights [8]. Generally, the full connection layer in the convolutional neural network will keep minimum to ensure that reduce the computational load.

\section{SVM of CNN}

The use of Support Vector Machine (SVM) in computer vision is mainly due to its good performance in the field of linear classification. SVM has good performance for basic binary classification. SVM is often used in the last few levels of convolutional neural networks. The SVM classifies and judges the feature vectors extracted by the front part of the convolutional neural network. SVM mainly relies on the inner product operation. It belongs to the generalized linear classifier. Its characteristic is that it can optimize the minimum error and the maximum interval at the same time, so it shows good performance in feature vector classification [9].

\section{DISCUSSION OF APPLICATION}

$\mathrm{CNN}$ is an algorithm widely used for image classification in the field of deep learning. This paper proposes to construct a convolutional neural network framework on the server side of the browser or website for the identification of some illegal images. It can also be used to update the database in real time. Not only for illegal websites, can the model proposed in this paper also be used for filtering information such as spam. In terms of computational cost, it mainly depends on the server, so that the load on the local host is small. Large-scale image classification and other operations can also be performed on the server side. CNN can also be used for speech recognition and classification.

\section{CONCLUSION}

This paper introduced convolutional neural network algorithm as the identification algorithm of illegal network to build server-side framework. This paper first made a basic introduction to the current method of identification of illegal networks, and then explained the structure and function characteristics of the introduced convolutional neural network algorithm. The convolutional neural network has good performance in image recognition, because of the too many parameters that need to be set in the early stage; there are also problems with the computational cost. However, with the continuous optimization of the CNN structure, it is believed that such problems can be resolved quickly.

\section{REFERENCES}

1. Tao Chun. (2017). In the smart age, security issues face new threats. China Education Network, (10), 5-6.

2. Wang $\mathrm{Na}$, Duan Pengfei, \& Wu Linlin. (2016). Identification of illegally operated vehicles based on convolutional neural networks. Computer Applications, 36(A02), 193-196.

3. Wu Yanfeng. (2016). Research and implementation of real-time face recognition system based on OpenCV (Doctoral dissertation, North China Electric Power University).

4. Hubel, D. H., \& Wiesel, T. N. (1962). Receptive fields, binocular interaction and functional architecture in the cat's visual cortex. The Journal of physiology, 160(1), 106-154.

5. Fukushima, K., \& Miyake, S. (1982). Neocognitron: A self-organizing neural network model for a mechanism of visual pattern recognition. In Competition and cooperation in neural nets (pp. 267-285). Springer, Berlin, Heidelberg.

6. Lacuna, Y., Bottom, L., Bagnio, Y., \& Heffner, P. (1998). Gradient-based learning applied to document recognition. Proceedings of the IEEE, 86(11), 2278-2324.

7. Karpathy, A. (2016). Cs231n convolutional neural networks for visual recognition. Neural networks, 1.

8. Karpathy, A., Toderici, G., Shetty, S., Leung, T., Sukthankar, R., \& Fei-Fei, L. (2014). Large-scale video classification with convolutional neural networks. In Proceedings of the IEEE conference on Computer Vision and Pattern Recognition (pp. 1725-1732).

9. Meyer, D., Leisch, F., \& Hornik, K. (2003). The support vector machine under test. Neurocomputing, 55(1-2), $169-186$. 